\section{BUPRENORPHINE: NOT A SILVER BULLET, AND STILL CONTROVERSIAL}

To the editor:

We are practitioners of pain medicine and addiction medicine and also four of the seven members of the Multi-Society Ad Hoc Substance Use Disorder (SUD) Working Group comprised of representatives from anesthesia, pain, pharmacy, and addiction medicine societies. We are finalizing "tip sheets" and a consensus-based manuscript to provide guidance on the appropriate use and initiation of buprenorphine in the hospital setting by anesthesiologists, and in the outpatient setting by pain clinicians.

While we wholeheartedly support the use of buprenorphine for pain and/or opioid use disorder (OUD) in appropriate clinical situations, we are concerned regarding the growing misconception among medical practitioners that buprenorphine should be used routinely as a first-line treatment for pain management and for all patients with pain and OUD. We wish to caution our colleagues: both chronic pain and/or SUDs are clinically complex conditions that must be addressed in a multimodal and often multidisciplinary fashion to move the needle in a positive direction. There simply is (to this point) no magic bullet to solve either condition. Buprenorphine is an opioid and in and of itself has inherent abuse potential. ${ }^{1}$ The unintended consequences of using buprenorphine as a first-line pain therapy could be significant and devastating, as we face another surge in the opioid epidemic during the COVID-19 pandemic, which has seen a significant rise in overdose deaths. ${ }^{2}$

Pharmacologically, buprenorphine has several benefits when compared to other opioids, including an improved side effect profile, kappa-antagonism to assist with mood symptoms, and a ceiling effect with regard to respiratory depression, of which all opioid prescribers live in fear. ${ }^{3-5}$ There is also a growing body of evidence that buprenorphine, despite its partial agonist effect at the mu receptor, exerts analgesic effect by its unique mechanism of binding to all three opioid receptors. ${ }^{6-9}$ These effects do result in reasonable downstream analgesia. ${ }^{10}$ It is also extremely effective for the treatment of symptoms of opioid withdrawal.
We welcome the increased interest in the use of buprenorphine +/- naloxone for treatment of OUD, as evidence for medication management for OUD care is strong ${ }^{11,12}$ and access to specialty SUD care is limited. According to 2019 data, only about 10 percent of those age 12 and over with a suspected substance use disorder received specialty treatment in the past year. ${ }^{13}$ That said, the efficacy of buprenorphine for OUD is similar to that of naltrexone. ${ }^{14}$ One must weigh all considerations in terms of choosing the appropriate treatment for patients, including severity of SUD, compliance with treatment, and concomitant medications, among others.

Despite its unique mechanism, buprenorphine is still an opioid that works at the mu receptor. According to at least one study, buprenorphine is one of the most widely diverted opioids. ${ }^{1}$ Its abuse potential remains high, even if a buprenorphine overdose is less likely to be fatal. As with all opioids, when it is combined with central nervous system depressants such as benzodiazepines, buprenorphine can increase the risk of death. ${ }^{15}$ Severe sedation and nausea have been reported with its use. Failure to heed proper instructions related to its use can result in precipitated withdrawal with symptoms of high severity due to its extremely high binding affinity compared to other opioids. Also, it remains unclear whether or not buprenorphine is as effective of an analgesic as full agonists. ${ }^{16,17}$ As with full opioid agonists, there is no long-term evidence suggesting that, for pain management, buprenorphine results in significantly improved functional outcomes. As buprenorphine is metabolized by CYP3A4, inhibitors of this particular enzyme can result in substantially increased effective dose and duration of action. ${ }^{3}$

Utilizing buprenorphine in treating OUD has saved lives. While buprenorphine may have an improved side effect profile compared to other opioids, and while the liability related to prescribing it for pain may be somewhat lower versus other opioids, it is our firm belief that buprenorphine should not replace all full opioid agonist medications or be a first-line treatment strategy. As with any other clinical situation, the risks and benefits of all therapies should be considered. Multimodal pain care must be individualized and a plan discussed collaboratively with the patient and interdisciplinary team. 
We applaud the pain community for its increasing awareness of substance use disorders within our patient population and commitment to address the issue more effectively. We must also exercise caution and remember that buprenorphine, while an excellent treatment modality for appropriate patients and clinical scenarios, is ultimately just a medication-not a panacea.

Sudheer Potru, DO, FASAM Assistant Professor

Department of Anesthesiology Emory University School of Medicine Atlanta, Georgia

Michael Sprintz, DO, DFASAM Founder, Sprintz Center for Pain/Sprintz Center for Recovery Assistant Clinical Professor, Division of Geriatrics and Palliative Medicine University of Texas Health Science Center at Houston, Houston, Texas

Antje M. Barreveld, MD Assistant Professor, Department of Anesthesiology Tufts University School of Medicine Newton-Wellesley Hospital Newton, Massachusetts

Lynn Kohan, MD Associate Professor, Department of Anesthesiology University of Virginia School of Medicine Charlottesville, Virginia

\section{REFERENCES}

1. Chilcoat HD, Amick HR, Sherwood MR, et al.: Buprenorphine in the United States: Motives for abuse, misuse, and diversion. J Subst Abuse Treat. 2019; 104: 148-157. DOI:10.1016/j.jsat.2019.07.005.

2. Alter A, Yeager C, Analyst O: COVID-19 Impact on US National Overdose Crisis. Available at: http://www.odmap.org/ Content/docs/news/2020/ODMAP-Report-June-2020.pdf. Accessed March 17, 2021.

3. Davis MP, Pasternak G, Behm B: Treating Chronic Pain: An Overview of Clinical Studies Centered on the Buprenorphine Option. Drugs. 2018; 78(12): 1211-1228. DOI:10.1007/s40265018-0953-Z.

4. Stein C, Machelska H: Modulation of peripheral sensory neurons by the immune system: Implications for pain therapy.
Pharmacol Rev. 2011; 63(4): 860-881. DOI:10.1124/pr.110.003145.

5. Ahmadi J, Jahromi MS, Ehsaei Z: The effectiveness of different singly administered high doses of buprenorphine in reducing suicidal ideation in acutely depressed people with co-morbid opiate dependence: A randomized, double-blind, clinical trial. Trials. 2018; 19(1): 462. DOI:10.1186/s13063-018-2843-9.

6. Raffa RB, Haidery M, Huang H-M, et al.: The clinical analgesic efficacy of buprenorphine. J Clin Pharm Ther. 2014; 39(6): 577 583. DOI:10.1111/jcpt.12196.

7. Meske DS, Lawal OD, Elder H, et al.: Efficacy of opioids versus placebo in chronic pain: A systematic review and metaanalysis of enriched enrollment randomized withdrawal trials. $J$ Pain Res. 2018; 11: 923-934. DOI:10.2147/JPR.S160255.

8. Koppert W, Ihmsen H, Körber N, et al.: Different profiles of buprenorphine-induced analgesia and antihyperalgesia in a human pain model. Pain. 2005; 118(1-2): 15-22. DOI:10.1016/ j.pain.2005.06.030.

9. Silverman SM: Opioid induced hyperalgesia: Clinical implications for the pain practitioner. Pain Physician. 2009; 12(3): 679-684.

10. Webster L, Gudin J, Raffa RB, et al.: Understanding Buprenorphine for Use in Chronic Pain: Expert Opinion. Pain Med. 2020; 21(4): 714-723. DOI:10.1093/pm/pnz356.

11. Mattick RP, Breen C, Kimber J, et al.: Buprenorphine maintenance versus placebo or methadone maintenance for opioid dependence. Cochrane Database Syst Rev. 2014; (2): CD002207. DOI:10.1002/14651858.CD002207.pub4.

12. Evans E, Li L, Min J, et al.: Mortality among individuals accessing pharmacological treatment for opioid dependence in California, 2006-10. Addict. 2015; 110(6): 996-1005. DOI: 10.1111/add.12863.

13. Substance Abuse and Mental Health Services Administration: Key substance use and mental health indicators in the United States: Results from the 2019 National Survey on Drug Use and Health (HHS Publication No. PEP20-07-01-001, NSDUH Series H-55). Rockville, MD: Center for Behavioral Health Statistics and Quality, Substance Abuse and Mental Health Services Administration. 2020.

14. Lee JD, Nunes EV, Novo P, et al.: Comparative effectiveness of extended-release naltrexone versus buprenorphine-naloxone for opioid relapse prevention (X:BOT): A multicentre, open-label, randomised controlled trial. Lancet. 2018; 391(10118): 309-318. DOI:10.1016/S0140-6736(17)32812-X.

15. Park TW, Larochelle MR, Saitz R, et al.: Associations between prescribed benzodiazepines, overdose death and buprenorphine discontinuation among people receiving buprenorphine. Addict. 2020; 115(5): 924-932. DOI:10.1111/ add.14886.

16. Aiyer R, Gulati A, Gungor S, et al.: Treatment of Chronic Pain With Various Buprenorphine Formulations: A Systematic Review of Clinical Studies. Anesth Analg. 2018; 127(2): 529-538. DOI:10.1213/ANE.0000000000002718.

17. Cote J, Montgomery L: Sublingual buprenorphine as an analgesic in chronic pain: A systematic review. Pain Med. 2014; 15(7): 1171-1178. DOI:10.1111/pme.12386. 